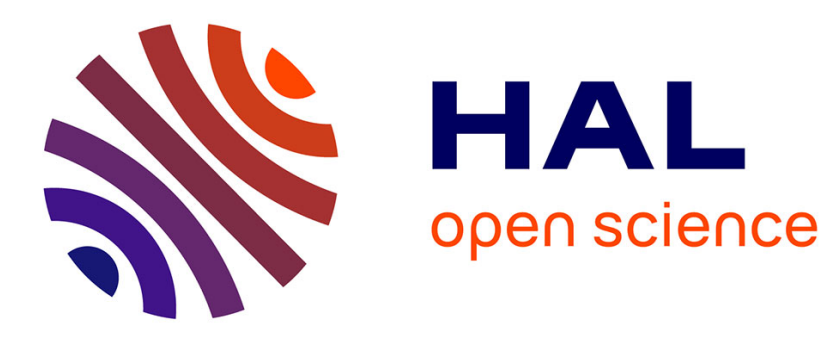

\title{
Conditions suffisantes de stabilité pour les solides visqueux
}

Farid Abed Meraim

\section{To cite this version:}

Farid Abed Meraim. Conditions suffisantes de stabilité pour les solides visqueux. Comptes Rendus de l'Academie des Sciences Serie II, 1999, 327, pp.25-31. 10.1016/S1287-4620(99)80006-4 . hal00111616

\section{HAL Id: hal-00111616 \\ https://hal.science/hal-00111616}

Submitted on 26 Aug 2019

HAL is a multi-disciplinary open access archive for the deposit and dissemination of scientific research documents, whether they are published or not. The documents may come from teaching and research institutions in France or abroad, or from public or private research centers.
L'archive ouverte pluridisciplinaire HAL, est destinée au dépôt et à la diffusion de documents scientifiques de niveau recherche, publiés ou non, émanant des établissements d'enseignement et de recherche français ou étrangers, des laboratoires publics ou privés.

\section{다)(1) $(5$}

Distributed under a Creative Commons Attribution - NonCommerciall 4.0 International 


\section{Conditions suffisantes de stabilité pour les solides visqueux}

\section{Farid ABED MERAIM}

Laboratoire de mécanique des solides, UMR CNRS 7649, École polytechnique, 91128 Palaiseau cedex, France

E-mail : abed@lms.polytechnique.fr

Résumé. Cette étude est consacrée à la stabilité de l'évolution quasi statique des solides et structures dépendant de la vitesse de déformation. Elle se distingue par sa capacité à répondre à la stabilité du problème non linéaire et pas simplement du problème linéarisé. La méthode de linéarisation des équations d'évolution non autonomes fournit une condition suffisante de stabilité. Cette condition, donnée par la définie positivité de la seconde variation de l'énergie libre totale, sera comparée au critère de nonbifurcation de Hill. Enfin, des exemples d'illustration en visco-élasticité et viscoplasticité sont présentés. (C) Académie des sciences/Elsevier, Paris

stabilité / bifurcation / visco-élasticité / visco-plasticité

\section{Sufficient conditions for stability of viscous solids}

Abstract. This study focuses on the stability of the quasi-static evolution of strain-rate-dependent solids and structures. This approach can deal with the stability of the non-linear problem, and not only with the linearised one. The linearisation method of nonautonomous evolution equations provides a sufficient stability condition. This condition given by the strict positiveness of the second derivative of the total free energy is compared to Hill's non-bifurcation criterion. Finally, some illustrative examples of visco-elasticity and visco-plasticity are presented. (c) Académie des sciences/Elsevier, Paris

stability / bifurcation / visco-elasticity / visco-plasticity

\section{Abridged English Version}

We consider here the stability analysis of the quasi-static evolution of strain-rate-dependent solids and structures. The main difficulty in studying the stability of such materials comes from the non-autonomous behaviour of the differential equations underlying their quasi-static evolution. We will use the stability notion in the sense of trajectory, first introduced by Lyapunov. Before proceeding, some fundamental results on the stability of the general non-autonomous differential equation (3) are given $[2,3]$. They provide to some extent a generalisation of Lyapunov's theorem in the nonautonomous case. 
In mechanical applications, this notion of stability gives a measure of the motion sensitivity to external perturbations. These perturbations may appear in many distinct forms: small displacement perturbations, or modification of some of the parameters involved in the differential equation (e.g. loading parameter). Assumed to be of finite duration, these perturbations can be reduced to a perturbation of the initial value. Therefore a viscous structure in the presence of imperfections falls into the scope of this study.

For the generalised standard materials [6], characterised by their free energy and dissipation potentials, the quasi-static evolution of a viscous solid in finite transformation is governed by equation (7). The linearisation of this differential equation of evolution around the fundamental solution $q^{0}(t)$ given by (8) provides us with sufficient stability conditions. In the case of a quadratic dissipation potential with strictly positive second derivative, the fundamental evolution $q^{0}(t)$ is uniformly and asymptotically stable if the second derivative of the total free energy is positive definite at every point of the evolution.

The elimination of the internal variables shows that this stability condition is equivalent to the strict positiveness of the limit hypo-elastic functional (9). This hypo-elastic functional corresponds to a particular non-linear elastic behaviour. The above-mentioned sufficient stability condition is generally more restrictive than Hill's non-bifurcation criterion for elasto-plastic solids [7]. This is due to the need for positiveness over a larger space of internal variables. However, it coincides with the latter criterion for systems with unidimensional stress and strain state, e.g., beams, rheological models, or monochrystals.

In particular, for Kelvin's visco-elastic model, (9) coincides with the second derivative of the total potential energy. In this case, the evolution $q^{0}(t)$ is stable if the elastic stability at the current points of this evolution is satisfied.

For non-quadratic dissipation potential or threshold (visco-plastic case) materials, the same approach leads to sufficient conditions of asymptotic stability (10) and (11). Condition (10) is generally more restrictive than (9). Functional (11) shows that under a sufficiently slow loading rate we obtain functional (9) again.

We illustrate our results by practical examples where Shanley's column [8], (figure 1) corresponding to three (visco-elastic and visco-plastic) types of behaviours is studied. For these three viscosity models corresponding to the Maxwell, Norton-Hoff, and Perzyna types respectively, we retrieve the same critical stability load given by $\lambda_{T}$ known as the critical load of the tangent modulus.

\section{Introduction}

Bon nombre de matériaux sont connus comme étant sensibles à la vitesse du chargement ; leur réponse à une sollicitation étant en partie différée dans le temps. L'absence d'équilibre pour de telles structures visqueuses nous suggère d'étudier la stabilité de leur évolution quasi statique. Cette notion de stabilité au sens des trajectoires conduit à des problèmes complexes car non autonomes. Il existe, en fait, très peu d'études sur ce sujet. Les quelques études existantes sont soit complètement linéarisées, ne répondant pas au problème non linéaire initial, soit postulant des solutions de forme exponentielle, ne tenant ainsi pas compte du caractère non autonome du problème. Aussi, notre approche se veut-elle globale, prolongeant les travaux, déjà existants, sur la stabilité des équilibres d'un solide en élastoplasticité [1]. 


\section{Résultats préliminaires de stabilité de systèmes non autonomes}

Considérons le système différentiel général non autonome suivant :

$$
\dot{y}=f(y, t)
$$

On suppose que pour toute condition initiale $y(0)=y_{0}$, il existe une solution unique $y(t),(t \geq 0)$ de l'équation (1). Soit $y^{0}(t)$ une solution particulière de l'équation (1) correspondant à une certaine condition initiale $y^{0}(0)$. Cette solution $y^{0}(t)$, dite fondamentale, est stable si pour toute perturbation $y^{*}\left(t_{0}\right)=y\left(t_{0}\right)-y^{0}\left(t_{0}\right)$ suffisamment faible appliquée au système à un instant $t_{0} \geq 0$ quelconque, la trajectoire perturbée $y(t)$ reste proche de la courbe fondamentale $y^{0}(t)$, pour toute valeur du temps $\left(t \geq t_{0}\right)$. En posant $y^{*}(t)=y(t)-y^{0}(t)$, l'équation différentielle vérifiée par $y^{*}$ est :

$$
\begin{aligned}
& \dot{y}^{*}=f\left(y^{*}+y^{0}(t), t\right)-f\left(y^{0}(t), t\right)=F\left(y^{*}, t\right) \quad\left(t \geq t_{0}\right) \\
& y^{*}\left(t_{0}\right)=y\left(t_{0}\right)-y^{0}\left(t_{0}\right) \quad \text { perturbation initiale donnée }
\end{aligned}
$$

La stabilité de la solution $y^{0}(t)$ de (1) est ainsi équivalente à la stabilité de la solution zéro de l'équation (2), au sens suivant: pour tout $\varepsilon>0$ et tout $t_{0} \geq 0$, il existe $\eta=\eta\left(\varepsilon, t_{0}\right)$ tel que $\left\|y^{*}\left(t_{0}\right)\right\|<\eta \Rightarrow\left\|y^{*}(t)\right\|<\varepsilon$ pour $t \in\left[t_{0},+\infty\left[\right.\right.$. Si, de plus, $\left\|y^{*}(t)\right\| \rightarrow 0$ lorsque $t \rightarrow+\infty$, la stabilité est dite asymptotique. Elle est dite uniforme si $\eta$ peut être choisi indépendamment de $t_{0}$.

Notons que des imperfections présentes dans une structure visqueuse sont un cas particulier de perturbation appliquée au système à $t=0$ et entrent ainsi dans le cadre de cette étude.

L'équation différentielle (1), modifiée par translation selon (2), s'écrit encore :

$$
\dot{y}^{*}=B(t) \cdot y^{*}+g\left(y^{*}, t\right)
$$

où $B(t)=\frac{\partial F}{\partial y^{*}}(0, t)=\frac{\partial f}{\partial y}\left(y^{0}(t), t\right)$ et la partie non linéaire $g\left(y^{*}, t\right)=F\left(y^{*}, t\right)-B(t) \cdot y^{*}$ supposée vérifier $\frac{\left\|g\left(y^{*}, t\right)\right\|}{\left\|y^{*}\right\|} \rightarrow 0$ lorsque $\left\|y^{*}\right\| \rightarrow 0$, uniformément par rapport à $t$.

La linéarisation de l'équation (1) sous la forme $\dot{y}^{*}=B(t) \cdot y^{*}$ peut donner des informations sur la stabilité de la solution fondamentale $y^{0}(t)$ de l'équation différentielle non linéaire de départ. On trouve dans la littérature $[2,3]$ les résultats suivants :

2.1. Proposition 1. - S'il existe un opérateur $G(t)$ borné, continûment dérivable et symétrique défini positif, tel que l'opérateur $\dot{G}(t)+B^{T} \cdot G+G \cdot B$ soit défini négatif pour tout $t$, alors la solution $y^{0}(t)$ de l'équation (1) est uniformément et asymptotiquement stable.

En prenant $G=I d$, la condition suffisante de stabilité précédente se réduit à la définie négativité de $B$ pour tout $t$. Ce qui équivaut à ce que toutes les valeurs propres de la partie symétrique de $B$ soient majorées uniformément par une constante strictement négative.

2. Proposition 2. - Si $B(t)=M^{-1}(t) \cdot K(t)$, avec $M$ symétrique définie positive, $K$ symétrique, alors si $\dot{M}+2 K(t)$ est définie négative pour tout $t$, la solution $y^{0}(t)$ de l'équation (1) est uniformément et asymptotiquement stable.

La condition suffisante de stabilité donnée ci-dessus est obtenue avec $G=M$. Si, de plus, $M$ est constante, elle se réduit alors à la simple définie négativité de $K$ pour tout $t$.

Pour l'exploitation de ces résultats dans le cadre de la mécanique, $M$ et $K$ seront remplacées formellement par les variations secondes de la dissipation et de l'énergie libre totale respectivement. Dans les conditions précédentes, toute petite perturbation appliquée au système à un instant $t_{0}$ 
quelconque décroît exponentiellement vers zéro lorsque $t$ croît à partir de $t_{0}$. Pour des systèmes mécaniques discrets, ces résultats de stabilité s'appliquent rigoureusement. Pour des milieux continus, les difficultés mathématiques liées aux choix des normes appropriées sont les mêmes qu'en théorie de stabilité élastique $[4,5]$.

\section{Solides visqueux en transformation finie}

On considère un solide visqueux quelconque en transformation finie, caractérisé dans le cadre des matériaux standard généralisés [6] par sa densité volumique d'énergie libre $W(\nabla u, \alpha)$ et son potentiel de dissipation $D(\nabla \dot{u}, \dot{\alpha})$. Les variables d'état $(\nabla u, \alpha)$ représentent le gradient lagrangien du déplacement et les variables internes. Les équations d'état et les lois complémentaires entre forces et flux sont données respectivement par (4) et (5) :

$$
\begin{array}{ll}
b^{R}=W, \nabla u & A=-W,_{\alpha} \\
b^{I R}=D, \nabla \dot{v}, & A=D,_{\alpha}
\end{array}
$$

où $b=b^{R}+b^{I R}$ est la décomposition du tenseur des contraintes de Piola-Lagrange ou de Boussinesq, en parties réversible et irréversible. En description lagrangienne, l'évolution quasi statique du solide est donnée par le principe des travaux virtuels :

$$
\int_{V} b: \nabla \delta u \mathrm{~d} v=\int_{\partial V_{T}} T(\dot{\lambda}) \cdot \delta u \mathrm{~d} s
$$

où $T(\lambda)$ représente les forces appliquées sur la partie $\partial V_{T}$ de la frontière et $\lambda(t)$ un paramètre de contrôle, $\lambda(t) \in\left[0, \hat{\lambda}_{M}\right]$.

Avec les notations: $q=(u, \alpha), \quad W(q, \lambda)=\int_{V} W(\nabla u, \alpha) \mathrm{d} v-\int_{\partial V_{T}} T(\lambda) \cdot u \mathrm{~d} s$, $\boldsymbol{D}(\dot{q})=\int_{V} D(\nabla \dot{u}, \dot{\alpha}) \mathrm{d} v$, l'évolution quasi statique se résume en une équation globale condensée :

$$
\left(\boldsymbol{W}_{q}+\boldsymbol{D},{ }_{\dot{q}}\right)(\delta q)=0 \quad \forall \delta q
$$

Cette équation différentielle (7) associe à un état initial $q(0)=(u(0), \alpha(0))$ donné une solution fondamentale $q^{0}(t)=\left(u^{0}(t), \alpha^{0}(t)\right)$, dont l'étude de stabilité est en question. La linéarisation de l'équation de l'évolution autour de $q^{0}(t)$ nous donne:

$$
\begin{aligned}
& \boldsymbol{W},_{q q}\left[\delta q, q^{*}\right]+\boldsymbol{D},_{q \dot{q}}\left[\delta q, \dot{q}^{*}\right]=0 \quad \forall \delta q \\
& \boldsymbol{W}_{,_{q q}}\left[\delta q, q^{*}\right]=\int_{V}\left[\nabla \delta u:\left(W,,_{\nabla \nabla}: \nabla u^{*}+W_{,_{\nabla u \alpha}}: \alpha^{*}\right)+\delta \alpha:\left(W_{,_{\alpha} \nabla u}: \nabla u^{*}+W_{,_{\alpha \alpha}}: \alpha^{*}\right)\right] \mathrm{d} v \\
& \boldsymbol{D},_{q \dot{q}}\left[\delta q, \dot{q}^{*}\right]=\int_{V}\left[\nabla \delta u:\left(D,,_{\nabla \dot{u} \nabla \dot{u}}: \nabla \dot{u}^{*}+D,_{\nabla \dot{u} \dot{\alpha}}: \dot{\alpha}^{*}\right)+\delta \alpha:\left(D, \dot{\alpha} \nabla \dot{u}: \nabla \dot{u}^{*}+D,_{\dot{\alpha} \dot{\alpha}}: \dot{\alpha}^{*}\right)\right] \mathrm{d} v
\end{aligned}
$$

Pour un potentiel de dissipation $D(\nabla \dot{u}, \dot{\alpha})$ quadratique, la forme bilinéaire symétrique $\boldsymbol{D},{ }_{q q}[\delta q, \delta q]$ est indépendante du temps $t$. Sous la condition de positivité stricte $\left(\boldsymbol{D},_{q q}[\delta q, \delta q] \geqslant c_{1}\|\delta q\|^{2}, \forall \delta q, c_{1}>0\right)$, le résultat suivant découle de la proposition 2 : 
3.1. Proposition 3. - Si la seconde variation de l'énergie libre totale du système est strictement positive $\left(\boldsymbol{W},{ }_{q q}\left(q^{0}(t)\right)[\delta q, \delta q] \geqslant c_{2}\|\delta q\|^{2}, \forall \delta q, c_{2}>0\right)$, en tout point de l'évolution, alors cette solution fondamentale $q^{0}(t)$ est uniformément et asymptotiquement stable.

Comme $\delta \alpha: W_{, \alpha}: \delta \alpha$ est strictement positif, on peut éliminer la variable $\alpha$ et la condition de stabilité (proposition 3) est équivalente à la définie positivité de la forme quadratique suivante :

$$
I(\delta u)=\frac{1}{2} \int_{V} \nabla \delta u:\left(W_{, \nabla u \nabla u}-W_{, \nabla u \alpha}: W_{, \alpha \alpha}^{-1}: W_{, \alpha \nabla u}\right): \nabla \delta u \mathrm{~d} v
$$

On appellera cette forme quadratique $I(\delta u)$, la fonctionnelle élastique limite. En effet, elle correspond à un comportement élastique non linéaire particulier, obtenu en écrivant que les vitesses

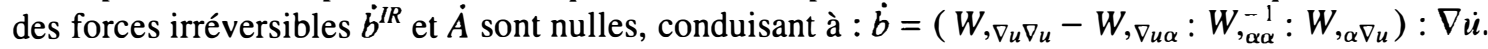

Dans le cas particulier d'un modèle de Kelvin, la fonctionnelle (9) s'identifie à la seconde variation de l'énergie potentielle totale. Dans ce cas, l'évolution $q^{0}(t)$ est stable si la condition de stabilité élastique aux points courants de cette évolution est satisfaite.

Ce critère de la fonctionnelle élastique limite (9) évoque fortement le critère de non-bifurcation de Hill [7] pour les solides élasto-plastiques. Dans le cas général, la condition (9) est plus restrictive que le critère de Hill car elle exige la définie positivité sur un espace de variation des variables internes plus grand. Cependant, on montre que pour les systèmes où l'état de contrainte et de déformation est unidimensionnel, cas des poutres, des modèles rhéologiques ou des monocristaux, la fonctionnelle élastique limite (9) s'identifie au critère de Hill.

Intéressons-nous maintenant à des matériaux à seuil ou à potentiel de dissipation non quadratique. On supposera, pour simplifier, que la déformation visco-plastique, notée $\alpha$, est le seul mécanisme dissipatif. Les équations d'équilibre et d'évolution précédentes restent valables, avec $D=D(\dot{\alpha})$, $\dot{\alpha}=\Omega,{ }_{A}$, où $\Omega(A)$ est le potentiel dual de dissipation. La linéarisation de l'équation d'évolution (7) conduit à la matrice non symétrique $\boldsymbol{B}$ écrite par blocs :

$$
\boldsymbol{B}=\left[\begin{array}{cc}
W, \nabla_{u} \nabla u & W, \nabla_{u \alpha} \\
\Omega,,_{A A}: W,_{\alpha \nabla u} & \Omega,_{A A}: W,_{\alpha \alpha}
\end{array}\right] ; \quad \boldsymbol{B}^{s}=\frac{1}{2}\left(\boldsymbol{B}+\boldsymbol{B}^{T}\right)
$$

Une condition suffisante de stabilité uniforme et asymptotique, d'après la proposition 1, est alors obtenue par symétrisation de $\boldsymbol{B}$. Elle exige la définie positivité de la fonctionnelle élastique limite $\boldsymbol{I}_{\boldsymbol{I}}$ ci-après, lorsque la matrice : $\Omega,_{A A}: W,_{\alpha \alpha}+W,_{\alpha \alpha}: \Omega,_{A A}$ est définie positive :

$$
\begin{gathered}
I_{1}(\delta u)=\frac{1}{2} \int_{V} \nabla \delta u:\left[W_{, \nabla u \nabla u}-\frac{1}{2}\left(W_{, \nabla u \alpha}+W_{, \nabla u \alpha}: \Omega,_{A A}\right):\left(\Omega,_{A A}: W_{,_{\alpha \alpha}}+W_{,_{\alpha \alpha}}: \Omega,_{A A}\right)^{-1}:\right. \\
\left.\left(W_{,_{\alpha \nabla u}}+\Omega_{,_{A A}}: W_{,_{\alpha \nabla u}}\right)\right]: \nabla \delta u \mathrm{~d} v
\end{gathered}
$$

Utilisant la proposition 2, une autre condition suffisante de stabilité asymptotique est donnée par la définie positivité de la forme quadratique: $\left(\boldsymbol{W}_{,_{q q}}-\frac{1}{2} \dot{\boldsymbol{D}}_{,_{q q}}\right)[\delta q, \delta q]$. Cette condition est aussi équivalente à la définie positivité de la fonctionnelle élastique limite $I_{2}$ suivante, lorsque la matrice : $W_{,_{\alpha \alpha}}-\frac{1}{2} \dot{D}_{\dot{\alpha} \dot{\alpha}}$ est définie positive :

$$
I_{2}(\delta u)=\frac{1}{2} \int_{V} \nabla \delta u:\left[W_{, \nabla u \nabla u}-W,,_{\nabla u \alpha}:\left(W_{,_{\alpha \alpha}}-\frac{1}{2} \dot{D}_{,_{\alpha \dot{\alpha}}}\right)^{-1}: W_{\alpha_{\alpha \nabla u}}\right]: \nabla \delta u \mathrm{~d} v
$$

Les conditions de stabilité portant sur $I_{1}(\delta u)$ et $I_{2}(\delta u)$ sont obtenues en exhibant des fonctions de Lyapunov pour la variable $\alpha$. La continuité de $u$ comme fonction de $\alpha$, sous la condition de stabilité élastique, montre alors que l'on a une stabilité asymptotique par rapport au couple $(u, \alpha)$. 
Notons que la condition de définie positivité de $\boldsymbol{I}_{1}(\delta u)$ est un peu plus restrictive que la définie positivité de $\boldsymbol{I}(\delta u)$ obtenue pour une dissipation quadratique. Cependant, pour bon nombre d'exemples usuels visco-plastiques étudiés, la condition (9) portant sur $I(\delta u)$ s'est révélée suffisante pour assurer la stabilité de l'évolution. Il sera montré plus généralement, dans une étude ultérieure, que la condition (9) est une condition suffisante de stabilité, quelle que soit la forme du potentiel de dissipation supposé convexe et indépendant de l'état actuel.

\section{Exemples d'illustration}

Pour illustrer ce qui précède, on va donner quelques exemples en visco-élasticité et en viscoplasticité. Considérons d'abord la colonne de Shanley $[8,9]$ discrète, constituée d'une tige rigide reposant sur deux ressorts visco-élastiques de type Maxwell à écrouissage cinématique (figure 1). On utilisera la notation vectorielle : $\boldsymbol{u}=(v, \theta), \boldsymbol{\alpha}=\left(\alpha_{1}, \alpha_{2}\right), \boldsymbol{\epsilon}(\boldsymbol{u})=\left(\epsilon_{1}, \epsilon_{2}\right)$ où : $\epsilon_{i}=v+(-1)^{l} l \sin \theta, i=1,2$. L'énergie potentielle totale du système s'obtient à partir du potentiel d'énergie libre $W(\boldsymbol{u}, \boldsymbol{\alpha})$ et du potentiel des efforts extérieurs $W_{\text {ext }}(\boldsymbol{u}, \lambda)$ et s'écrit, en gardant toutes les non-linéarités géométriques : $\mathbb{E}(\boldsymbol{u}, \boldsymbol{a}, \lambda)=\frac{1}{2} E(\boldsymbol{\epsilon}-\boldsymbol{a})^{T} \cdot(\boldsymbol{\epsilon}-\boldsymbol{a})+\frac{1}{2} h \boldsymbol{a}^{T} \cdot \boldsymbol{a}-\lambda[v+L(1-\cos \theta)]$. Les relations de comportement et d'évolution sont : $\boldsymbol{\sigma}=W,_{\epsilon}=E(\epsilon-\boldsymbol{\alpha}) ; \boldsymbol{A}=-W,_{\boldsymbol{a}}=\boldsymbol{\sigma}-h \boldsymbol{\alpha} ; \dot{\boldsymbol{a}}=\frac{1}{\eta} \boldsymbol{A}$. L'équilibre des forces et des moments s'exprime par le système : $\mathbb{E}_{,_{u}}=\mathbf{0} \Leftrightarrow\left(\mathbb{E}_{,_{v}}=0, \mathbb{E}_{, \ell}=0\right)$. La solution fondamentale $\left(\boldsymbol{u}^{0}(t), \boldsymbol{a}^{0}(t)\right)$, correspondant à $(\theta(t)=0)$, s'obtient complètement analytiquement pour un chargement $\lambda(t) \in\left[0, \lambda_{M}\right]$ par la résolution d'un problème de Cauchy. En linéarisant les équations d'évolution autour de la solution $\left(\boldsymbol{u}^{0}(t), \boldsymbol{\alpha}^{\boldsymbol{0}}(t)\right)$, on trouve :

$$
\left\{\begin{array}{l}
\mathbb{E}_{,_{\boldsymbol{u} u}} \cdot \boldsymbol{u}^{*}+\mathbb{E}_{\boldsymbol{u}_{\boldsymbol{u a}}} \cdot \boldsymbol{a}^{*}=\mathbf{0} \\
\dot{\boldsymbol{a}}^{*}=-\frac{1}{\eta}\left(\mathbb{E}_{,_{\boldsymbol{a a}}} \cdot \boldsymbol{a}^{*}+\mathbb{E}_{,_{\boldsymbol{a u}}} \cdot \boldsymbol{u}^{*}\right)
\end{array}\right.
$$

La matrice $\mathbb{E}_{,_{u u}}$ est inversible tant que l'on reste en dessous de la charge critique d'Euler : $\lambda_{E}=\frac{2 E l^{2}}{L}$,

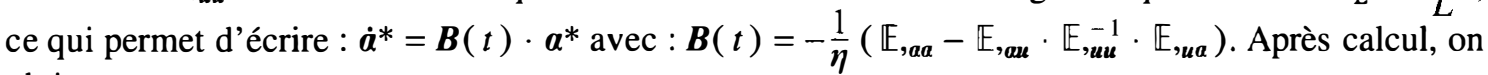
obtient :

$$
\boldsymbol{B}(t)=-\frac{1}{\eta}\left[\begin{array}{cc}
h-p & p \\
p & h-p
\end{array}\right], \quad \text { avec } \quad p=\frac{E \lambda}{2\left(\lambda_{E}-\lambda\right)}
$$

Les valeurs propres de cette matrice symétrique sont: $\mu_{1}=-\frac{h}{\eta}$ et $\mu_{2}=-\frac{h}{\eta} \frac{\lambda_{E}}{\lambda_{T}} \frac{\lambda_{T}-\lambda}{\lambda_{E}-\lambda}$ $\left(\lambda_{T}=\frac{2 E_{T} l^{2}}{L}, E_{T}=\frac{E h}{E+h}\right)$, associées respectivement aux vecteurs propres $(1,1)$ et $(1,-1)$.

On conclut donc, d'après la proposition 1 , que la solution fondamentale $\left(\boldsymbol{u}^{0}(t), \boldsymbol{a}^{0}(t)\right)$ est uniformément et asymptotiquement stable si $\lambda_{M}<\lambda_{T}$. Puisque le vecteur propre associé à $\mu_{2}$ est constant, on peut également affirmer que l'évolution quasi statique est instable pour $\lambda_{M}>\lambda_{T}$. Il vient alors qu'une condition nécessaire et suffisante de stabilité est donnée par la charge critique du module tangent $\lambda_{T}$.

Dans le cas d'un potentiel dual de dissipation $\Omega(\boldsymbol{A})$ non quadratique, par exemple de type Norton-Hoff, $\left(\Omega(\boldsymbol{A})=c\left[\left(\frac{A_{1}}{A_{c}}\right)^{m}+\left(\frac{A_{2}}{A_{c}}\right)^{m}\right], c>0, m:\right.$ paire), la règle d'écoulement devient : $\dot{\boldsymbol{a}}=\Omega,{ }_{\boldsymbol{A}}=c \frac{m}{A_{c}}\left(\frac{\boldsymbol{A}}{A_{c}}\right)^{m-1}$. Il convient alors de remplacer le coefficient $\frac{1}{\eta}$ de la matrice $\boldsymbol{B}(t)$ dans 
Figure 1. Colonne de Shanley visqueuse.

Figure 1. Viscous Shanley's column.

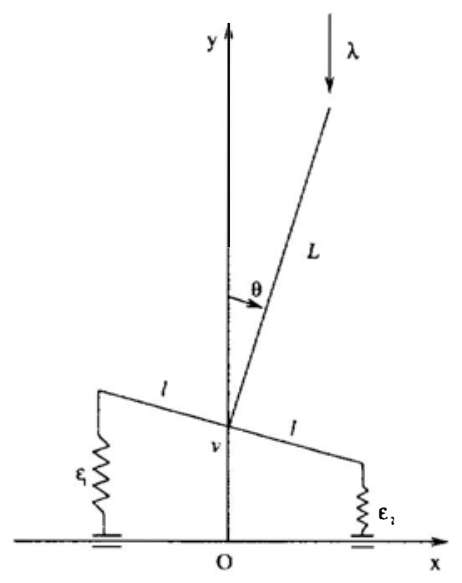

(12) $\operatorname{par} \Omega,_{A_{i} A_{i}}^{0}=c \frac{m(m-1)}{A_{c}^{2}}\left(\frac{A_{i}^{0}(t)}{c}\right)^{m-2}>0$. Les résultats de stabilité sont alors identiques. Notons que l'on retrouve le cas du modèle visco-élastique de Maxwell en prenant $m=2$ et $c=\frac{A_{c}^{2}}{2 \eta}$. Enfin, pour un matériau visco-plastique à seuil, le modèle de Perzyna par exemple consiste à prendre pour potentiel dual de dissipation : $\Omega(\boldsymbol{A})=\frac{1}{2 \eta}\left[<\left|A_{1}\right|-A_{c}>^{2}+<\left|A_{2}\right|-A_{c}>^{2}\right]$, où $<>$ désigne la partie positive. La loi d'évolution s'écrit : $\dot{\alpha}_{i}=\frac{1}{\eta}<\left|A_{i}\right|-A_{c}>$ signe $\left(A_{i}\right), i=1,2$. La solution fondamentale $\left(\boldsymbol{u}^{0}(t), \boldsymbol{a}^{0}(t)\right)$, correspondant à $\theta(t)=0$, se calcule alors explicitement pour un chargement croissant $\lambda(t) \in\left[0, \lambda_{M}\right]$. Elle contient une phase élastique suivie d'une partie viscoplastique. La linéarisation des équations de l'évolution, dans le domaine visco-plastique, donne la même relation (12) avec le même opérateur $\boldsymbol{B}(t)$. Les résultats de stabilité sont donc trouvés identiques au cas visco-élastique précédent.

Notons que dans les modèles de Norton-Hoff et de Perzyna, on retrouve un comportement élastoplastique à écrouissage cinématique lorsque l'on fait tendre les paramètres $\frac{1}{m}$ respectivement $\eta$ vers zéro.

\section{Références bibliographiques}

11] Nguyen Q.S., Radenkovic D., Stability of equilibrium in elastic plastic solids, Proc. IUTAM-IMU Symposium, Marseille, 1975, On Applications of Fonctionals to Problems of Mechanics, lecture note in Math. 503, Springer-Verlag, Berlin, 1976.

|2| Roseau M., Vibrations non linéaires et théorie de la stabilité, Springer-Verlag, Berlin, 1966, vol. 8.

[3] Hahn W., Stability of Motion, Springer-Verlag, Berlin, 1967.

14] Koiter W.T., A basic open problem in the theory of elastic stability, Proc. IUTAM-IMU Symposium, Marseille, 1975, On Applications of Fonctionals to Problems of Mechanics, lecture note in Math. 503, Springer-Verlag, Berlin, 1976.

[5] Potier-Ferry M., Critères de l'énergie en élasticité et visco-élasticité. Le flambement des structures, Éditions du bâtiment et des travaux publics, Paris, 1981.

[6] Halphen B., Nguyen Q.S., Sur les matériaux standard généralisés, J. Mech. 14 (1) (1975) 39-63.

[7] Hill R., A general theory of uniqueness and stability in elastic-plastic solids, J. Mech. Phys. Solids 6 (1958) $236-249$.

[8] Shanley F.R., Inelastic column theory, J. Aero. Sci. 14 (1947) 261-267.

[9] Abed Meraim F., Sur quelques problèmes de stabilité et de bifurcation des solides visqueux, thèse de doctorat, École polytechnique, 1999, $250 \mathrm{pp}$. 